\title{
Aqueous Affinity and Interfacial Dynamics of Anisotropic Buckled Black Phosphorous
}

\section{Adyasa Priyadarsini and Bhabani S. Mallik*}

Department of Chemistry, Indian Institute of Technology Hyderabad, Sangareddy-502285, Telangana, India

*bhabani@chy.iith.ac.in

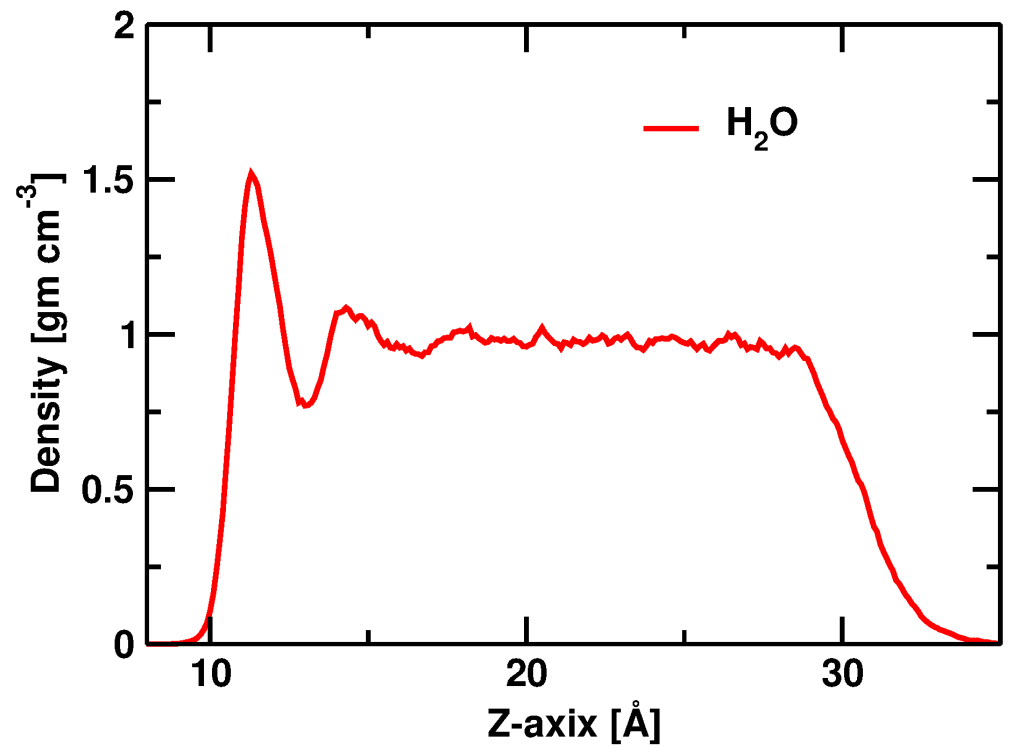

Figure S1. The density profile of water (red line) along the z-direction of the simulation box.

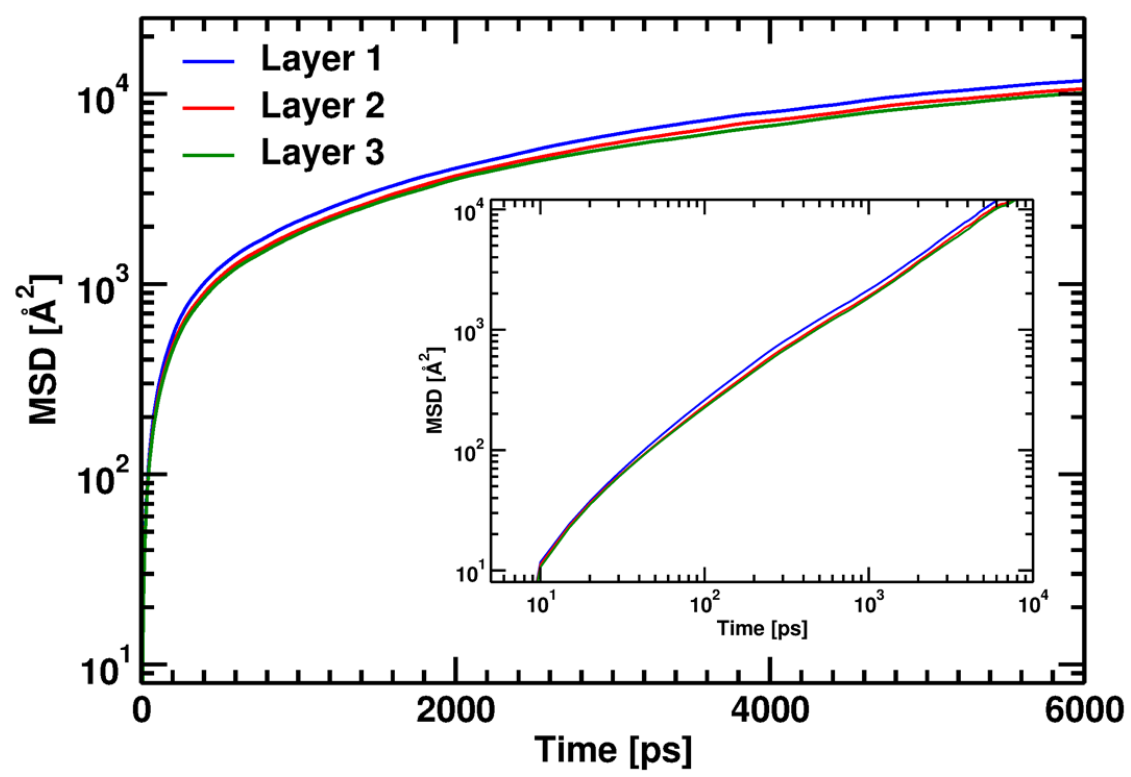

Figure S2. The semi-log plot of mean squared displacement (MSD) of water in the layer 1, 2 and 3 represented by the bule, red and green lines, respectively. The log-log plot is provided in the snippet. 


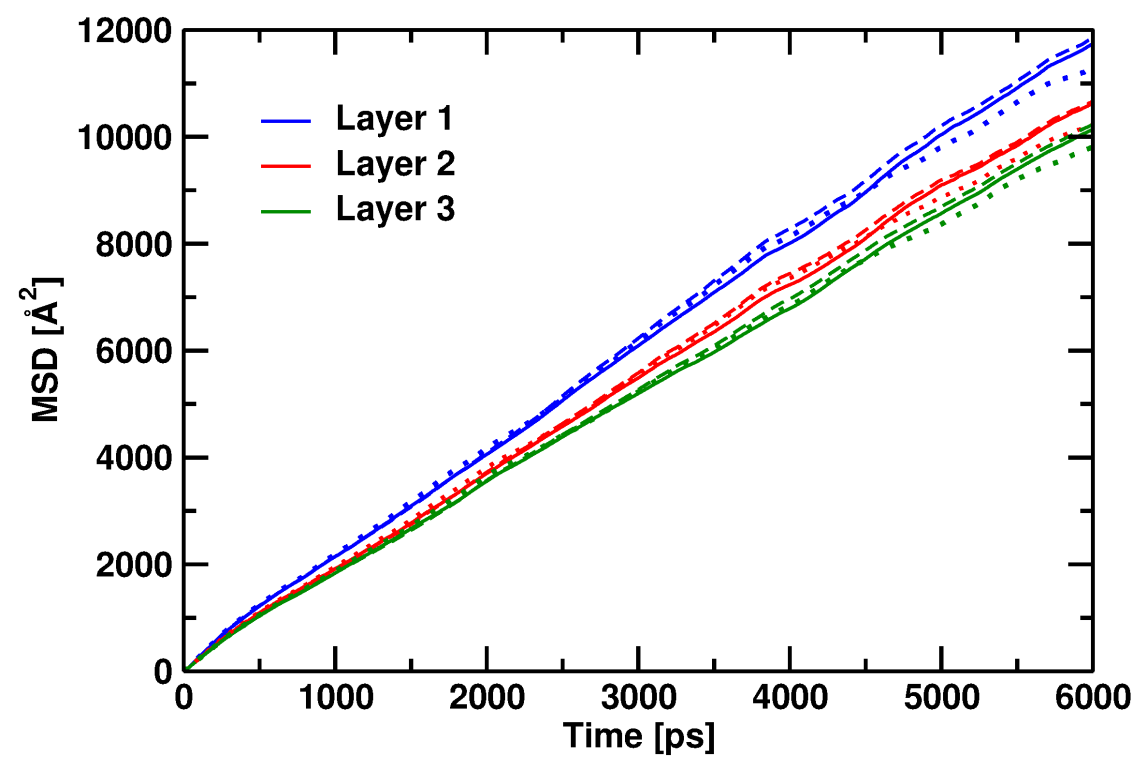

Figure S3. Mean squared displacement (MSD) of water molecules in layer 1 (blue line), layer 2 (red line), and layer 3/bulk (green line). The solid, dash, and dot line represents three different sets of calculations performed on L1, L2, and L3 water molecules.

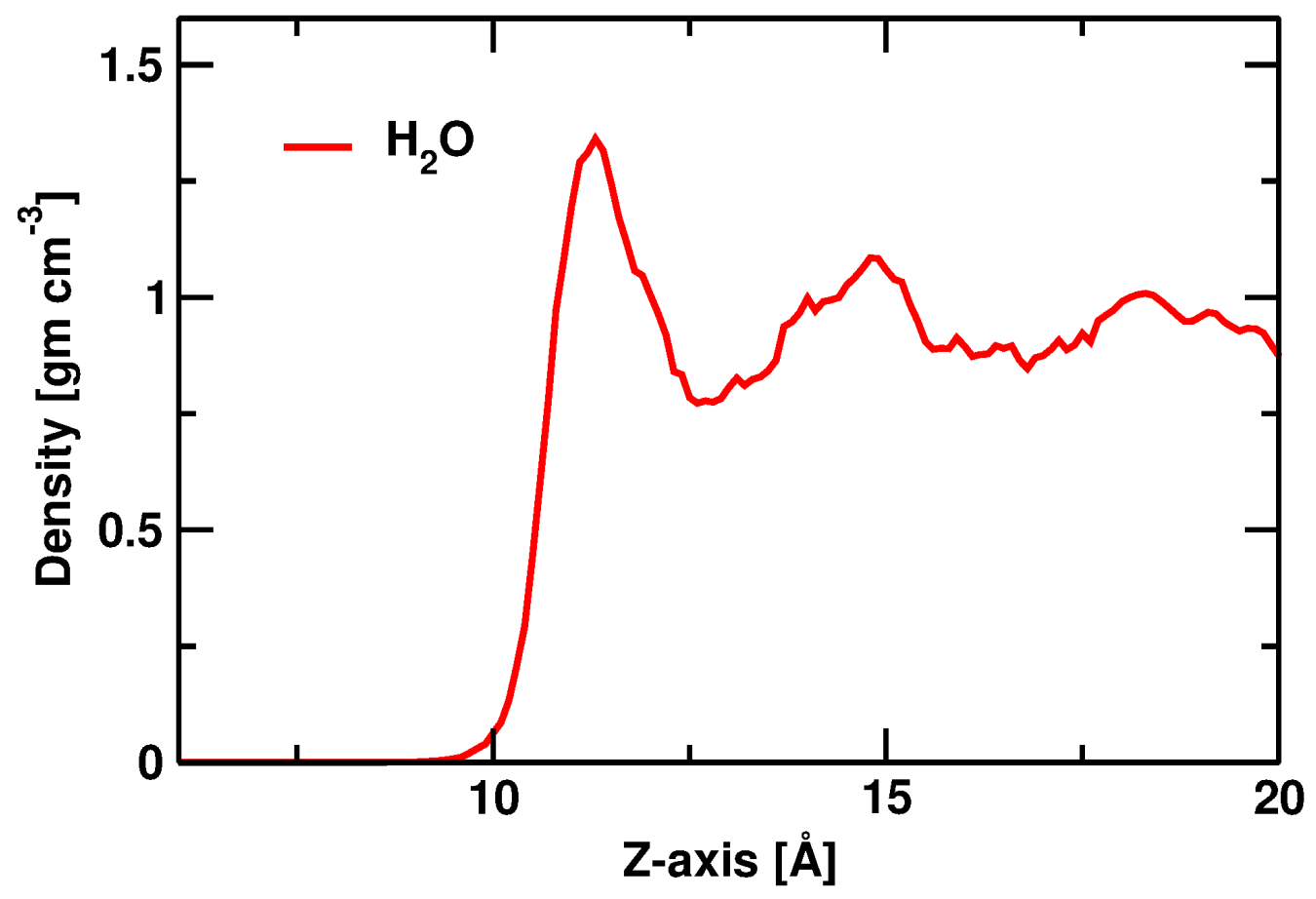

Figure S4. The density profile of water (red line) along the z-direction of the simulation box, obtained from the first principles molecular dynamics simulations. 